Article

\title{
First Description of the Mitogenome and Phylogeny of Culicinae Species from the Amazon Region
}

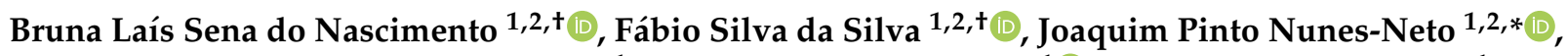 \\ Daniele Barbosa de Almeida Medeiros ${ }^{1}$, Ana Cecília Ribeiro Cruz ${ }^{1}{ }^{\infty}$, Sandro Patroca da Silva ${ }^{1}$, \\ Lucas Henrique da Silva e Silva ${ }^{2}$, Hamilton Antônio de Oliveira Monteiro ${ }^{1}$, Daniel Damous Dias ${ }^{2}$, \\ Durval Bertram Rodrigues Vieira ${ }^{1}$, José Wilson Rosa, Jr. ${ }^{1}$, Roberto Carlos Feitosa Brandão ${ }^{1}$, \\ Jannifer Oliveira Chiang ${ }^{1}$, Livia Carício Martins ${ }^{1}{ }^{1}$ and Pedro Fernando da Costa Vasconcelos ${ }^{2} \mathbb{B}$
}

1 Department of Arbovirology and Hemorrhagic Fevers, Ananindeua, Evandro Chagas Institute-IEC/SVS/MS, Belém 67030-000, Brazil; brunanascimento@iec.gov.br (B.L.S.d.N.); fabiodasilva@iec.gov.br (F.S.d.S.); danielemedeiros@iec.gov.br (D.B.d.A.M.); anacecilia@iec.gov.br (A.C.R.C.); sandrosilva@iec.gov.br (S.P.d.S.); hamiltonmonteiro@iec.gov.br (H.A.d.O.M.); durvalvieira@iec.gov.br (D.B.R.V.); josej@@iec.gov.br (J.W.R.J.); robertobrandao@iec.gov.br (R.C.F.B.); janniferchiang@iec.gov.br (J.O.C.); liviamartins@iec.gov.br (L.C.M.)

2 Biological and Health Sciences Center, Parasitary Biology in the Amazon Posgraduation Program, State of Pará University, Belém 66095-662, Brazil; lucashenriqueuepa@gmail.com (L.H.d.S.e.S.); damous1994@gmail.com (D.D.D.); pedrovasconcelos@iec.gov.br (P.F.d.C.V.)

Citation: do Nascimento, B.L.S.; da Silva, F.S.; Nunes-Neto, J.P.; de Almeida Medeiros, D.B.; Cruz, A.C.R.; da Silva, S.P.; da Silva e Silva, L.H.; de Oliveira Monteiro, H.A.; Dias, D.D.; Vieira, D.B.R.; et al. First Description of the Mitogenome and Phylogeny of Culicinae Species from the Amazon Region. Genes 2021, 12, 1983. https:// doi.org/10.3390/genes12121983

Academic Editors: Maria Anice Mureb Sallum and Jan E. Conn

Received: 12 November 2021 Accepted: 1 December 2021

Published: 14 December 2021

Publisher's Note: MDPI stays neutral with regard to jurisdictional claims in published maps and institutional affiliations.

Copyright: (c) 2021 by the authors. Licensee MDPI, Basel, Switzerland. This article is an open access article distributed under the terms and conditions of the Creative Commons Attribution (CC BY) license (https:// creativecommons.org/licenses/by/ $4.0 /)$.
* Correspondence: joaquimneto@iec.gov.br

$\dagger$ Equal first authors.

Abstract: The Culicidae family is distributed worldwide and comprises about 3587 species subdivided into the subfamilies Anophelinae and Culicinae. This is the first description of complete mitochondrial DNA sequences from Aedes fluviatilis, Aedeomyia squamipennis, Coquillettidia nigricans, Psorophora albipes, and Psorophora ferox. The mitogenomes showed an average length of 15,046 pb and $78.02 \%$ AT content, comprising 37 functional subunits (13 protein coding genes, 22 tRNAs, and two rRNAs). The most common start codons were ATT/ATG, and TAA was the stop codon for all PCGs. The tRNAs had the typical leaf clover structure, except $t R N A^{\text {Ser } 1}$. Phylogeny was inferred by analyzing the 13 PCGs concatenated nucleotide sequences of 48 mitogenomes. Maximum likelihood and Bayesian inference analysis placed Ps. albipes and Ps. ferox in the Janthinosoma group, like the accepted classification of Psorophora genus. Ae. fluviatilis was placed in the Aedini tribe, but was revealed to be more related to the Haemagogus genus, a result that may have been hampered by the poor sampling of Aedes sequences. Cq. nigricans clustered with Cq. chrysonotum, both related to Mansonia. Ae. squamipennis was placed as the most external lineage of the Culicinae subfamily. The yielded topology supports the concept of monophyly of all groups and ratifies the current taxonomic classification.

Keywords: Culicidae; mitochondrial genome; sequencing; molecular taxonomy

\section{Introduction}

The Family Culicidae (Meigen, 1818), known as mosquitoes, is a large taxon distributed worldwide. The Culicidae comprises about 3587 officially recognized species, classified in the subfamilies Anophelinae and Culicinae, accepted to be monophyletic despite some inner relations that are not entirely understood [1]. This family of insects is responsible for transmitting the etiologic agents of several arboviruses such as dengue virus, yellow fever virus, Chikungunya virus, and Zika virus, filariosis from Wuchereria bancrofti and malaria, caused by Plasmodium spp. [2].

The Subfamily Anophelinae includes the genera Anopheles, Bironella and Chagasia, while the subfamily Culicinae includes a variety of genera within the tribes Aedeomyiini, Aedini, Culicini, Culisetini, Ficalbiini, Hoggesiini, Mansoniini, Orthopodomyiini, Sabethini, 
Toxorhynchitini, and Uranotaeniini, totalizing 110 genera [1-4]. The Anophelinae subfamily is demonstrably monophyletic, a basal lineage in relation to the Culicidae family. To date, 493 species are recorded, with many complex and sibling-species relationships awaiting deeper phylogenetic and taxonomic resolution, mainly in the Anopheles genus [1,5,6]. The unknown medical/veterinary importance of Bironella and Chagasia makes most of the studies focus towards the genus Anopheles due its capacity to transmit malaria parasites, in addition to several arboviruses $[7,8]$. The monophyletic status for Bironella is not supported in most of the studies [6,9], but the opposite is found to the genus Anopheles, specifically the subgenus Stethomyia, Lophopodomyia, Kerteszia and Cellia [9].

Like the Anophelinae, the phylogenetic history of the Culicinae subfamily is not completely settled, but some groups have been better studied due their epidemiological role as potential vectors of microorganisms. Former studies in the Sabethini tribe had investigated its phylogenetic relationships mainly through morphological and biogeographical aspects, and despite the fact the Sabethini was considered to be monophyletic, such cladistics analysis displayed some divergences among the genera, notably in the Wyeomyia genus recovered as a large paraphyletic taxa [2,10]. More recent mitogenome studies reiterated the monophyly of neotropical Sabethini species corroborating Reidenbach's findings based on both morphological and molecular data [11,12]. The Culicini tribe has grounded monophyletic status, but there are many cryptic species that hamper the natural classification of the group, mainly in the Culex genus [13-15]. The Aedini tribe is undoubtedly the most critical group in terms of phylogenetic reconstruction, it is an enormous group where over time, dozens of subgenera rose to genera status followed by restoration, and until now there is no phylogeny consensus. In a broader view, the tribe is treated as monophyletic and encompassing three genera (traditional classification: Aedes, Haemagogus and Psorophora), but its interrelations lack deeper and wider analysis, especially for the genus Aedes [1-4].

In order to improve taxonomic and evolutionary studies, the use of genomic tools has increasingly contributed significant information, for example, for the development of vector control strategies or population variability assessments [16-18]. In this context, one of the genomic markers most currently used is the mitochondrial genome (mitogenome), which consists of a compact double-stranded circular molecule, with a length ranging from 15 to $20 \mathrm{~kb}$, housing 37 functional subunits, 13 of which are active in protein coding (PCGs), 22 regions of transporter RNA (tRNA) and two ribosomal RNA regions, in addition to a terminal region rich in adenine and thymine $(\mathrm{A}+\mathrm{T})$, associated with replicative processes of the molecule [19]. The use of this genomic set is considered advantageous for the absence of introns, the relative low gene recombination and for its maternal haploid inheritance. For the past few years, the use of the mitogenome has been producing relevant results in the development of evolutionary investigations for a wide variety of groups of organisms, ranging from insects to mammals, but in particular arthropods of medical-epidemiological importance [5,20-26].

Despite the great number of Culicidae of medical importance, particularly in the context of the Brazilian Amazon region, there is in contrast no availability of genomic information in public data repositories of these organisms with few exceptions, and considering the great potential of the applicability of molecular markers such as the mitochondrial genome in the development of investigations of mosquito molecular taxonomy, we present in this study, for the first time, the characterization of the mitogenome of the species Aedeomyia squamipennis Lynch Arribálzaga, 1878, Aedes (Grc.) fluviatilis Lutz, 1904, Coquillettidia (Rhy.) nigricans Coquillet, 1904, Psorophora (Jan.) albipes Theobald, 1907, and Psorophora (Jan.) ferox Humboldt, 1819, all occurring in the Brazilian Amazon, using high throughput sequencing (HTS) and evolutionary considerations.

\section{Materials and Methods}

\subsection{Sample Collection and Total DNA Extraction}

The mosquito species investigated in this study derived from several ecoepidemiological investigations realized by the Department of Arbovirology and Haemorrhagic Fevers 
of Evandro Chagas Institute (IEC/SVS/MS). Sample collection were performed by the human attraction method during field expeditions in the municipalities of Altamira, Canaã dos Carajás, Santa Bárbara and Belém (Combu Island), in the state of Pará, carried out between 2017 and 2019 (Table 1).

Table 1. Collection information on the evaluated species.

\begin{tabular}{|c|c|c|c|c|c|c|c|c|}
\hline Species & ID-Code & Collected & Used & Sex & Collection Site & State & $\begin{array}{c}\text { Geographical } \\
\text { Coordinates }\end{array}$ & Year \\
\hline Ad. squamipennis & AR_867100 & 6 & 5 & q & Altamira & Pará & $-3.203333,-52.206389$ & 2017 \\
\hline Ae. fluviatilis & AR_866516 & 13 & 5 & q & Canaã dos Carajás & Pará & $-6.496944,-49.878333$ & 2019 \\
\hline Cq. nigricans & AR_850512 & 45 & 5 & 우 & Altamira & Pará & $-3.203333,-52.206389$ & 2017 \\
\hline Ps. albipes & AR_859743 & 30 & 5 & q & Belém & Pará & $-1.495795,-48.454260$ & 2017 \\
\hline Ps. ferox & AR_849712 & 14 & 5 & q & Santa Bárbara & Pará & $-1.209000,-48.271722$ & 2017 \\
\hline
\end{tabular}

The authorization to carry out activities allowing the collection of biological samples of mosquitoes was granted by the Biodiversity Information and Authorization System of the Ministry of Environment (SISBIO/MMA) under registration numbers 56004-1 and 56504-5. The specimens collected were identified based on external morphology characteristics with dichotomous keys for representatives of the subfamily Culicinae [4], later the identification was confirmed by comparison with DNA barcode sequences available on GenBank (Table 2). The specimens were organized in groups containing up to 45 individuals belonging to the same species, following storage at $-70{ }^{\circ} \mathrm{C}$ for further procedures. Following the taxonomic identification process, five mosquitoes were removed from each species-group to compose the biological sample to be sequenced. The samples were macerated individually (per species) using $3 \mathrm{~mm}$ stainless steel spheres in a TissueLyser II device (Qiagen, Hilden, ME, Germany), and soon after, total DNA was extracted using a commercial DNeasy Blood \& Tissue kit (Qiagen, Hilden, ME, Germany), following the recommendations of the manufacturer.

Table 2. Taxonomic confirmation of evaluated species based on the barcode sequence of COI genes. Access codes from species presented below may be consulted on the NCBI database.

\begin{tabular}{cccccc}
\hline $\begin{array}{c}\text { Species } \\
\text { Evaluated }\end{array}$ & \multicolumn{5}{c}{ Nucleotide Identity (\%)-COI (Barcode DNA) } \\
\hline & $\begin{array}{c}\text { Ad. squamipennis } \\
\text { AF417729 }\end{array}$ & $\begin{array}{c}\text { Oc. fluviatilis } \\
\text { KF314736 }\end{array}$ & $\begin{array}{c}\text { Cq. nigricans } \\
\text { KM593033 }\end{array}$ & $\begin{array}{c}\text { Ps. albipes } \\
\text { MN997524 }\end{array}$ & $\begin{array}{c}\text { Ps. ferox } \\
\text { MN997516 }\end{array}$ \\
\hline $\begin{array}{c}\text { Ad. } \\
\text { squamipennis }\end{array}$ & 98.99 & - & - & - & - \\
Ae. fluviatilis & - & 98.78 & - & - & - \\
Cq. nigricans & - & - & 99.85 & - & - \\
Ps. albipes & - & - & - & 95.14 & - \\
Ps. ferox & - & - & - & - & 99.7 \\
\hline
\end{tabular}

\subsection{Quantification of Total Extracted DNA, Construction of the Genomic Library, and Sequencing}

The total DNA extracted from each lot of identified species was quantified using the Qubit 4.0 fluorometer equipment (Life Technologies, Carlsbad, CA, USA) with the commercial Qubit dsDNA Hs Assay kit (Invitrogen, Waltham, MA, USA), following the recommendations of the manufacturers. The total DNA products extracted from each batch were previously standardized at a concentration of $0.2 \mathrm{ng} / \mu \mathrm{L}$, and then fragmented and labeled with adapter sequences (i7 and i5) following the recommendations of the Nextera XT DNA library preparation (Illumina, San Diego, CA, USA) protocol. The labeled DNA fragments were then purified using the Agencourt AMPure XP kit (Beckman Coulter, Brea, CA, USA). After completion of the previous steps, the genomic libraries obtained were quantified using the Qubit 4.0 fluorometer (Life Technologies, Carlsbad, CA, USA), and their quality was checked using a High Sensitivity DNA kit (Agilent Technologies, Santa 
Clara, CA, USA), following the recommendations established by the manufacturer. Finally, the products obtained were sequenced using the NextSeq 500/550 High Output Kit and in a run of 150 cycles $(2 \times 75)$ NextSeq500 platform (Illumina, San Diego, CA, USA).

\subsection{Pre-Processing of Sequenced Products}

At the end of the genomic sequencing reaction, the data generated in Illumina Base Call (.bcl) format were transferred from the NextSeq 500/550 platform equipment to a computer workstation, where the analysis and genomic characterization steps were performed. Files in ".bcl" format were converted to ".fastq" format using bcl2fastq Conversion v.2.20 (Illumina, San Diego, CA, USA) software. The FastQC v.0.11.9 software [27] was used to previously verify the quality metrics of the data obtained, and then, the software Trim Galore v.0.6.5 [28] was used for the removal of adapter sequences. At the end of this process, a new quality check and validation of the data obtained was performed using FastQC.

\subsection{Genomic Assembly}

The pre-processed sequencing files of each species investigated in this study were submitted to a genomic assembly step by the de novo method using the software MEGAHIT v.1.2.9 [29] and SPAdes v.3.15.2 [30]. The contigs generated and corresponding to the mitochondrial readings of each species evaluated were selected using the DIAMOND software [31], and visualized using the MEGAN6 software [32]. The assembled contigs corresponding to the mitochondrial genome of each species were compared to genomic data from reference sequences (Aedes albopictus Genbank ID: NC_006817, and Coquillettidia chrysonotum Genbank ID: NC_044655), and were manually inspected using Geneious v.11.0 software [33].

\subsection{Analyses of the Obtained Mitochondrial Sequences}

The online MITOchondrial genome annotation Server (MITOS—http:/ / mitos.bioinf. uni-leipzig.de/index.py, accessed on 1 November 2021) tool [34] was used for automatic annotation and prediction of secondary tRNA structures of the mitochondrial sequences obtained in this study. The graphic maps containing the representations of the mitochondrial genomes obtained were generated using the CGView software [35], and the nucleotide composition metrics of each sequence were obtained using the Geneious v.11.0 and MEGAX software [36]. Based on the nucleotide composition metrics obtained, the composition biases based on the AT and GC asymmetry values were estimated using the formulas: AT-skew $=(\mathrm{A} \%-\mathrm{T} \%) /(\mathrm{A} \%+\mathrm{T} \%)$ and GC-skew $=(\mathrm{G} \%-\mathrm{C} \%) /(\mathrm{G} \%+\mathrm{C} \%)[37]$ In addition, the relative use of synonymous codons (RSCU) was estimated based on the set of all 13 PCGs, using MEGAX. A sliding window of $200 \mathrm{bp}$ in steps $25 \mathrm{bp}$ was performed using the PopGenome package [38] in the statistical software R [39], and the pairwise comparison of the proportions of non-synonymous $(d N)$ and synonymous $(d S)$ substitutions $(d N / d S)$ among the obtained sequences were calculated using the CodeML software, part of the PAML package [40]. For plotting in graphs the results obtained based on nucleotide composition metrics, AT/GC skews, RSCU biases, and $d N / d S$ ratios, the statistical software $\mathrm{R}$ was used ( $\mathrm{R}$ Foundation for Statistical Computing, Vienna, Austria. Available in: https:/ /www.R-project.org/, accessed on 1 November 2021).

\subsection{Phylogenetics Analysis}

Using the Geneious v.11.0 software, all 13 PCGs of each of the sequences obtained in this study were extracted together, as well as other taxa available in the Genbank-NCBI and EMBL data repositories, being aligned using the MAFFT algorithm [41]. Nucleotide distances between the sequences used were obtained using the MEGAX software, based on the maximum composite likelihood method. The reconstruction of the phylogenetic relationships between the analyzed species was based on two different methodologies: for the definition of the best nucleotide substitution model according (having been defined the GTR+F+R4 model) to the Akaike information criterion (AIC) and the phylogeny recon- 
struction using the maximum likelihood methodology, the IQtree v.1.6.12 software [42] was used with bootstraping values defined for 1000 repetitions; and for the reconstruction of the phylogeny using the Bayesian inference methodology, the MrBayes v3.2.7a software [43] was used, with the performance of two simultaneous and independent reactions, with four chains each (three hot chains and a cold chain), defined for 1,000,000 generations, with sampling of the topologies generated every 1000 steps, and with a relative burn of $25 \%$. Finally, the obtained topologies were visualized using Figtree v.1.4.4 software [44], and edited using Inkscape [45].

\section{Results and Discussion}

\subsection{Organization, Structure and Content of the Mitogenomes Obtained}

The mitogenome of Ad. squamipennis, Cq. nigricans, Ae. fluviatilis, Ps. albipes, and Ps. ferox (Tables S1-S5), were arranged into classical compact circular double-strand DNA molecules, counting 37 functional and conserved subunits: 13 PCGs, 22 tRNAs, and two rRNA, organized along the J (forward) and R (reverse) strands (Figure 1).

The size of the sequences obtained ranged from 14,789 bp (Cq. nigricans) to 15,307 bp (Ps. ferox), and the overall AT content ranged from $77.5 \%$ (Ae fluviatilis) to $78.9 \%$ (Cq. nigricans). Considering the sets of PCGs, tRNAs and rRNAs, the species presented, respectively, average AT compositions of $76.6 \%, 79.7 \%$, and $82.3 \%$ (Figure $2 \mathrm{~A}$, Table 3 ). These results are similar to the putative characteristics from previous reported mosquito mitogenomes. The mitogenome of insects in general displays some particular features like the highly conserved genome architecture, low GC content and codon usage bias, such characteristics were recorded in this study $[5,19,22,23,46]$.

Additionally, the analysis of the composition asymmetry for each mitogenome resulted in positive values for AT-skews and negative values for CG-skews (Figure 2B,C, Table 3), meaning the greater proportion of adenine and cytosine on the majority chain compared to the thymine and guanine ratio. These findings resemble the results of previous studies that observed a nucleotide composition pattern for Culicidae [5,22,23,46-48], and other insect groups $[24,26,49,50]$.

All five of the mitogenomes presented a set of 22 tRNAs each. The classical cloverleaflike structure, with four arms [amino acid acceptor (AA), dihydrouridine (DHU), TYC, and anticodons (AC)] and loops [AA, DHU, T $\Psi C$, and variable (V)] were retrieved by secondary structure prediction analysis from 21 of the 22 subunits. The remaining unit $t R N A^{\operatorname{Ser} 1}$ shows a notable feature of reduced DHU arm, replaced by a loop. Same features reported in earlier research with Culicidae and other insects [5,22,23,46,47,49-52]. The tRNAs had an average length of $1484 \mathrm{bp}$ and average overall AT content of 79.7\%. Furthermore, 15 mismatched pair bases were detected for Ae. fluviatilis (13 GU and two UU pairs), 19 mismatches were identified for $A d$. squamipennis (16 GU and three UU pairs), 20 mismatches for Ps. ferox (19 GU and one UU), 24 mismatches recorded for both Cq. nigricans (21 GU and three UU), and Ps. albipes (23 GU and one UU) (Figures S1-S5).

Regarding only the PCGs subunits, the lengths ranged from $11,170 \mathrm{bp}$ of Ad. squamipennis (AT\% content of 76.7\%) to 11,357 bp of Cq. nigricans (AT\% content of $77.8 \%$ ). All PCGs had negative values for AT-skews, except for subunit ATP8 in Ae. fluviatilis and Ad. squamipennis (Figure 2B). Likewise, GC-skews were often negative, except for the subunits ND5, ND4, ND4L and ND1 for all sequences (Figure 2C). All the PCGs stop codon for the five species was TAA and the most frequent start codon were ATT/ATG. The AT content increased substantially in the third position, up to 93\% in Ps. albipes (in Cq. nigricans, 92.5\%; in Ad. squamipennis, 91.7\%; in Ps. ferox, 91.4\%; and in Ae. fluviatilis, 89.2\%) (Tables S1-S5). 

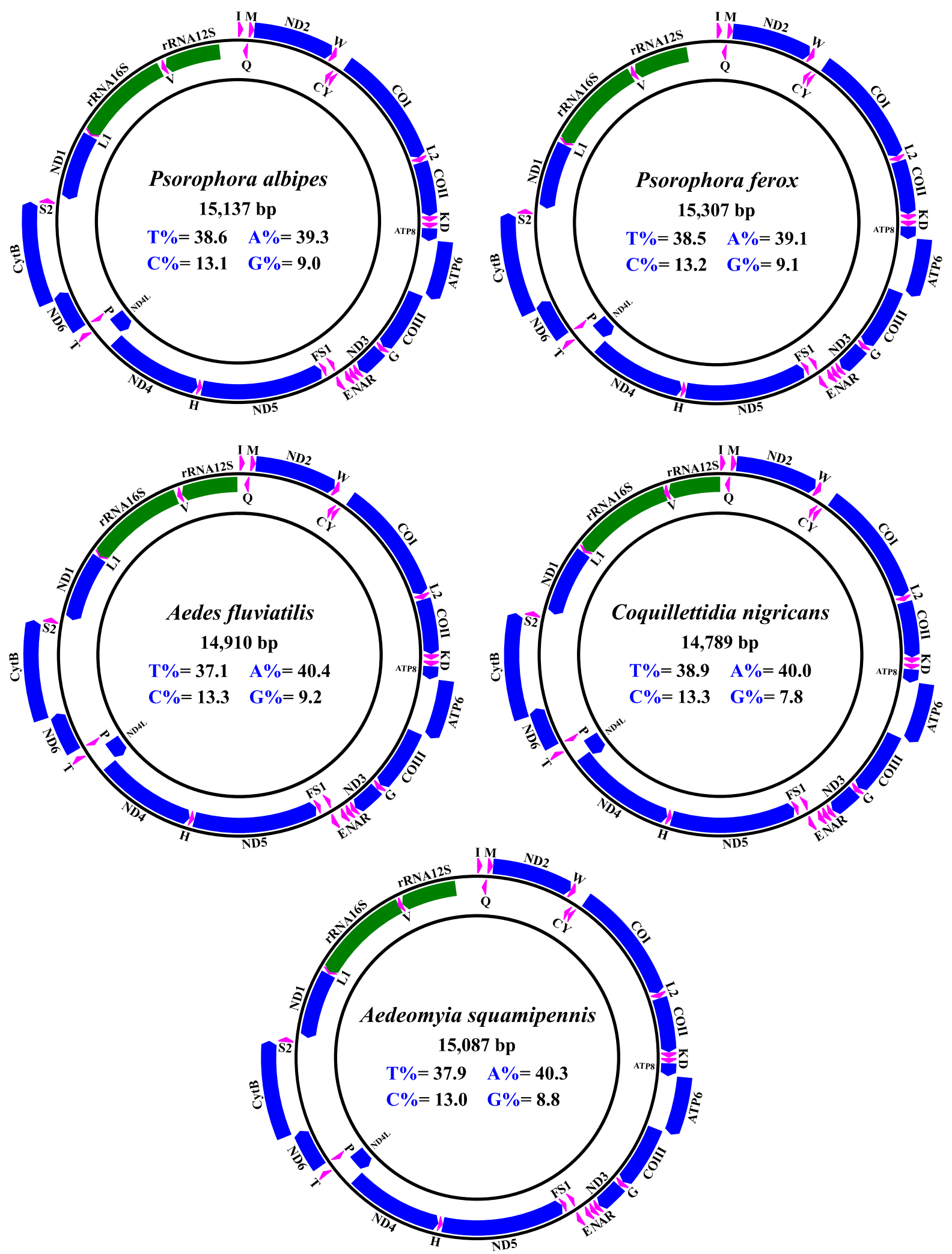

Figure 1. Structural representations of the Ps. albipes, Ps. ferox, Ae. fluviatilis, Cq. nigricans and Ad. squamipennis mitogenomes. The internal values indicate the overall content of the nucleotide bases. The blue, pink, and green blocks indicate PCGs, tRNAs, and rRNAs, respectively. Each tRNA is identified by a unique letter abbreviation. The genes arranged in the outer circle are located in the J strand (Forward), and the genes arranged in the inner circle are located in the $\mathrm{N}$ strand (Reverse). 

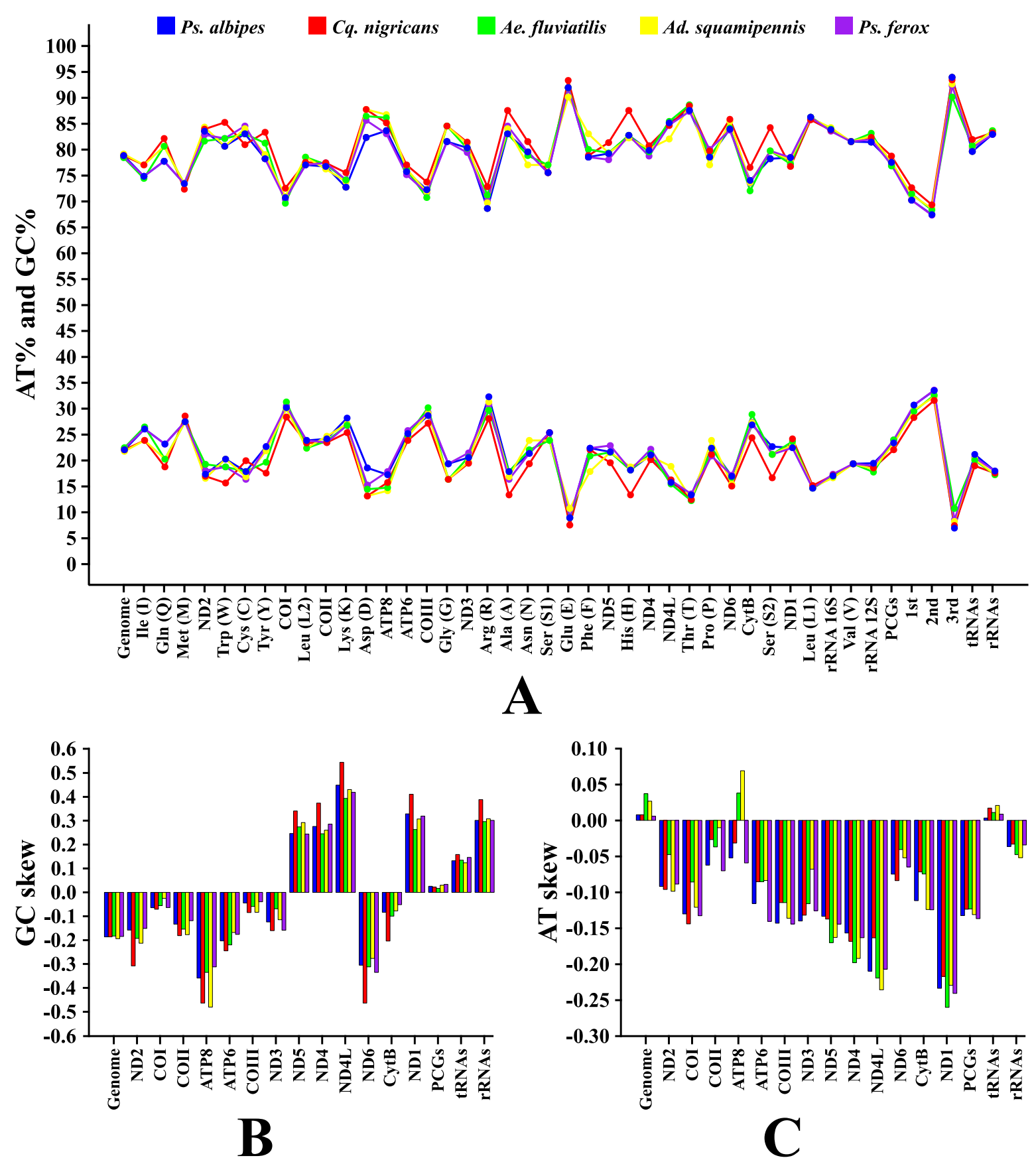

Figure 2. Information on AT/GC contents, and AT/GC-skews of the investigated mitogenomes. (A) AT contents (\%). (B) AT-skews. (C) GC-skews. 
Table 3. General metrics of the mitogenomes obtained in this study.

\begin{tabular}{|c|c|c|c|c|c|c|c|c|}
\hline Species & Size (bp) & $\mathbf{A} \%$ & $\mathrm{~T} \%$ & $\mathrm{C} \%$ & $\mathrm{G} \%$ & $\mathrm{AT} \%$ & AT-Skew & GC-Skew \\
\hline \multicolumn{9}{|l|}{ Whole mitogenome } \\
\hline Aedeomyia squamipennis & 15,087 & 40.3 & 37.9 & 13.0 & 8.8 & 78.2 & 0.0307 & -0.1927 \\
\hline Aedes fluviatilis & 14,910 & 40.4 & 37.1 & 13.3 & 9.2 & 77.5 & 0.0426 & -0.1822 \\
\hline Coquillettidia nigricans & 14,789 & 40.0 & 38.9 & 13.3 & 7.8 & 78.9 & 0.0139 & -0.2607 \\
\hline Psorophora albipes & 15,137 & 39.3 & 38.6 & 13.1 & 9.0 & 77.9 & 0.0090 & -0.1855 \\
\hline $\begin{array}{l}\text { Psorophora ferox } \\
\text { PCGs }\end{array}$ & 15,307 & 39.1 & 38.5 & 13.2 & 9.1 & 77.6 & 0.0068 & -0.1839 \\
\hline Aedeomyia squamipennis & 11,170 & 32.6 & 44.1 & 11.3 & 12.0 & 76.7 & -0.1499 & 0.0300 \\
\hline Aedes fluviatilis & 11,216 & 32.6 & 43.3 & 11.8 & 12.2 & 75.9 & -0.1410 & 0.0167 \\
\hline Coquillettidia nigricans & 11,357 & 33.4 & 44.4 & 10.8 & 11.3 & 77.8 & -0.1414 & 0.0226 \\
\hline Psorophora albipes & 11,269 & 32.5 & 44.1 & 11.4 & 12.0 & 76.6 & -0.1514 & 0.0256 \\
\hline $\begin{array}{l}\text { Psorophora ferox } \\
\text { tRNAs }\end{array}$ & 11,270 & 32.1 & 44.0 & 11.6 & 12.4 & 76.1 & -0.1564 & 0.0333 \\
\hline Aedeomyia squamipennis & 1470 & 40.8 & 38.9 & 8.9 & 11.4 & 79.7 & 0.0238 & 0.1232 \\
\hline Aedes fluviatilis & 1486 & 40.4 & 39.4 & 8.7 & 11.4 & 79.8 & 0.0125 & 0.1343 \\
\hline Coquillettidia nigricans & 1479 & 41.3 & 39.7 & 8.0 & 11.0 & 81.0 & 0.0198 & 0.1579 \\
\hline Psorophora albipes & 1493 & 39.5 & 39.2 & 9.2 & 12.0 & 78.7 & 0.0038 & 0.1321 \\
\hline $\begin{array}{l}\text { Psorophora ferox } \\
\text { rRNAs }\end{array}$ & 1494 & 40.1 & 39.3 & 8.8 & 11.8 & 79.4 & 0.0101 & 0.1456 \\
\hline Aedeomyia squamipennis & 2160 & 38.9 & 43.8 & 6.0 & 11.3 & 82.7 & -0.0593 & 0.3064 \\
\hline Aedes fluviatilis & 2151 & 39.1 & 43.6 & 6.1 & 11.2 & 82.7 & -0.0544 & 0.2948 \\
\hline Coquillettidia nigricans & 2097 & 39.6 & 42.7 & 5.4 & 12.2 & 82.3 & -0.0377 & 0.3864 \\
\hline Psorophora albipes & 2154 & 39.3 & 42.7 & 6.3 & 11.7 & 82.0 & -0.0415 & 0.3000 \\
\hline Psorophora ferox & 2160 & 39.4 & 42.6 & 6.3 & 11.7 & 82.0 & -0.0390 & 0.3000 \\
\hline
\end{tabular}

The subunits recognized as the putative $r R N A^{16 \mathrm{~S}}$ and $r R N A^{12 \mathrm{~S}}$ when concatenated ranged from $2097 \mathrm{bp}$ (Cq. nigricans) to 2160 (Ad. squamipennis and Ps. ferox). The AT content were actually very similar, despite almost all species being from different genera (average of $82.4 \%$ ), also, in the five mitogenomes obtained global AT-skews were negative and GC-skews positive (Figure 2B,C). Individually, the larger $r R N A^{16 S}$ counted $1376 \mathrm{bp}$ (Cq. nigricans) and the shorter $1359 \mathrm{bp}$ (Ps. albipes). For $r R N A^{12 S}$ the larger subunit counted $795 \mathrm{bp}$ (Ps. albipes and Ps. ferox) and the shorter $721 \mathrm{bp}$ (Cq. nigricans). As already reported previously, in the five sequences the subunit $r R N A^{16 \mathrm{~S}}$ was flanked by the $t \mathrm{RNA}^{\text {Leu1 }}$ and $t$ RNA ${ }^{V a l}$ and $r R N A^{12 S}$ was flanked by the same $t \mathrm{RNA}^{\mathrm{Val}}$ and the control region, the last non-sequenced. Such values are in agreement with earlier mosquito mitogenome studies $[5,22,23,48]$.

The sequences obtained presented between eight and 15 small intergenic and noncoding regions, with lengths ranging from one bp to $34 \mathrm{bp}$, thus totaling in Ps. albipes 105 non-coding bp; in Cq. nigricans, 108 bp; in Ae. fluviatilis, 124 bp; in Ad. squamipennis, $65 \mathrm{bp}$; and in Ps. ferox, 101 bp. Previous studies related such small intergenic spaces, and it was thought to be related to occurrence of overlapping reading frames and accordingly compact genome structure, such as the mitogenome $[23,53]$.

No data for $\mathrm{A}+\mathrm{T}$ region control were obtained in this study from any of the five species. As broadly known, this region is naturally rich in homopolymers, which constitutes an impairment for next generation sequencers. Furthermore, in invertebrates this particular region is highly variable in terms of length and rates of mutations (significant and nonsignificant). Similar to the presented results, this region was not obtained in other studies either. Lemos et al. [54] and Silva et al. [23], in their studies on the characterization of the mitogenome of species of the genus Haemagogus, did not recover this region from the obtained sequences. In order to improve the sequencing assays, alternative methods can be used to fully fill this lacuna such as conventional PCR and Sanger's sequencing method, once they allow target amplification, as already proved in similar studies [55-58].

\subsection{Description of Protein-Coding Genes (PCGs)}

For all the five mitogenomes obtained, nine PCGs displayed sense of transcription on the forward strand (J): ATP6, ATP8, COI, COII, COIII, CytB, ND2, ND3, and ND6, and the remaining genes showed sense of transcription on the reverse strand (N): ND5, ND4, 
NDL4, and ND1. A variety of start codons were used, present in the five sequences: ATT, ATG, TTG, and GTG (except in Cq. nigricans). Other start codons were also detected such as ATC (Ae. fluviatilis, Cq. nigricans, and Ps. ferox) and ATA (Ad. squamipennis), and all genes used TAA as standard stop codon (Tables S1-S5). Same arrangement and composition were previously described in other studies with Culicidae mitogenome [5,22,23,47].

In the genes ND2, COI, and ND3, for all species the start codon recorded was ATT, the same start codon is displayed in $A T P 8, N D 6$, and CytB from Ps. albipes; also for ND4 and $C y t B$ from $C q$. nigricans; ND3 and CytB from Ae. fluviatilis, ATP8 and ND6 from $A d$. squamipennis; and ATP8 and CytB from Ps. ferox sequence. The ATG start codon was recorded from COIII and ND4L for all species, additionally this codon also signals encoding for COII and ND4 from Ps. albipes and Ps. ferox, COII and ND5 from Cq. nigricans, COII and ATP6 from Ae. fluviatillis and ATP6, ND4 and CytB from Ad. squamipennis mitogenome. The TTG start codon was retrieved from the ND1 gene for all species, and individually for ATP6 from $C q$. nigricans, $P$ s. albipes, and Ps. ferox. The GTG start codon signaling process for the ND5 gene in all species, except for $C q$. nigricans. The ATC start codon was recorded for ATP8 and ND6 for both Cq. nigricans and Ae. fluviatilis, and ND6 from Ps. ferox, and finally the ATA start codon was recorded only for COII from Ad. squamipennis. No incomplete stop codons(T/TA) were found in any of the sequences (Tables S1-S5), although this is a relatively common event in Culicidae and other insects [22,23,46,47].

Disregarding the stop codons, there are a total of 3667 codons in use in the $A d$. squamipennis mitogenome, followed by 3665 in Ae. fluviatilis, 3651 in Cq. nigricans, and 3650 in the Ps. albipes and Ps. ferox mitogenome. The relative synonymous codon usage (RSCU) showed that nearly all codons seem to be used on the five sequences, however the AGG (S) codon was not expressed at all. Furthermore, CUG (L) was expressed only in the Ae. fluviatilis mitogenome, and Ps. albipes did not express ACC (T) and CGC (R), likewise Ps. ferox did not express UCG (S) and CCG (P) (Figure 3). The RSCU analysis also showed that codons with adenine and/or thymine (uracil) in the latter position were highly expressed in contrast with other synonymous codons that express cytosine and/or guanine in the third position, the NNA/NNU RSCU value were often above 1 (Table S6).

Leucine (L) synthesized from the UUA codon was the most frequent amino acid in the five species (Figure 3), representing 5.09 on RSCU analysis for Ps. albipes, followed by 5.02 in Ps. ferox, 4.98 in both Ae. fluviatilis and Ad. squamipennis, and 4.97 in Cq. nigricans, further codons most frequently used were: arginine in CGA (R) type (average RSCU value 2.95) and serine in UCU (S) type (2.87). Leucine in the CUC type was the least frequent in Ps. albipes mitogenome (RSCU $=0.02$ ), along with threonine $(\mathrm{T})$ in ACG type and glycine in GGC type (RSCU = 0.02). The same leucine $(C U C)$ was also the least frequent amino acid in Ps. ferox mitogenome (RSCU $=0.02)$, followed by glutamine in GAG $(\mathrm{E})$ type $(\mathrm{RSCU}=0.03)$. Threonine (ACG) was the least frequent codon in Cq. nigricans mitogenome (RSCU $=0.02)$ followed by proline in CCG type (RSCU = 0.03). Leucine in CUG and CUC type were the least expressed amino acids in Ae. fluviatilis (RSCU $=0.01$ and 0.02 , respectively). The CCG proline $(\mathrm{P})$ had the lowest amino acid frequency in the Ad. squamipennis sequence, representing the RSCU value of 0.03 (CCG codon). In all sequences, the type codons NNC and NNG had RSCU value $<1$, as observed in other studies of mitogenome sequencing of other mosquito and insect species [22,23,47,48,59-61].

\subsection{Evolutive Analysis}

In order to verify the evolutionary pressure acting on the different regions of the protein-coding mitogenomes obtained, based on the observation of ratios established between the non-synonymous and synonymous substitution rates $(d N / d S)$, paired analyses were performed between the sequences corresponding to each PCG of the investigated species, with results plotted in two different graphical presentation models. The results obtained indicated that the different regions evaluated are evolving globally under the effect of negative (or purifying) pressure, with $d N / d S$ values $<1$ (Figure $4 \mathrm{~A}, \mathrm{~B}$ ), with ratios ranging from $0.0208 \pm 0.0835$ in COI to $0.0010 \pm 0.5603$ in $A T P 8$, and thus, presenting 
the following order of influence of the purifying evolutionary pressure according to the averages obtained: $\mathrm{COI}<\mathrm{CytB}<\mathrm{COIII}<\mathrm{COII}<\mathrm{ATP} 6<\mathrm{ND} 1<\mathrm{ND} 3<\mathrm{ND} 2<\mathrm{ND} 5<\mathrm{ND} 4 \mathrm{~L}$ $<N D 6<N D 4<A T P 8$ (Figure 4B). Additionally, as reported in other studies, it was observed that the purifying evolutionary pressure was particularly stronger on PCGs belonging to mitochondrial complexes III (CytB) and IV (COI, COII, and COIII), in contrast to the regions of complex I $(N A D H)$, which, like $A T P 8$, although with $d N / d S$ rates $<1$, showed less conservative evolutionary pressure restrictions, with relaxed purifying pressure and brief signs of positive evolutionary pressure (adaptive). The results obtained in this analysis still corroborate the pattern of heterogeneity between the evolutionary rates, although purifying, acting on different PCGs despite the taxonomic distance among the evaluated species $[5,22,23]$.
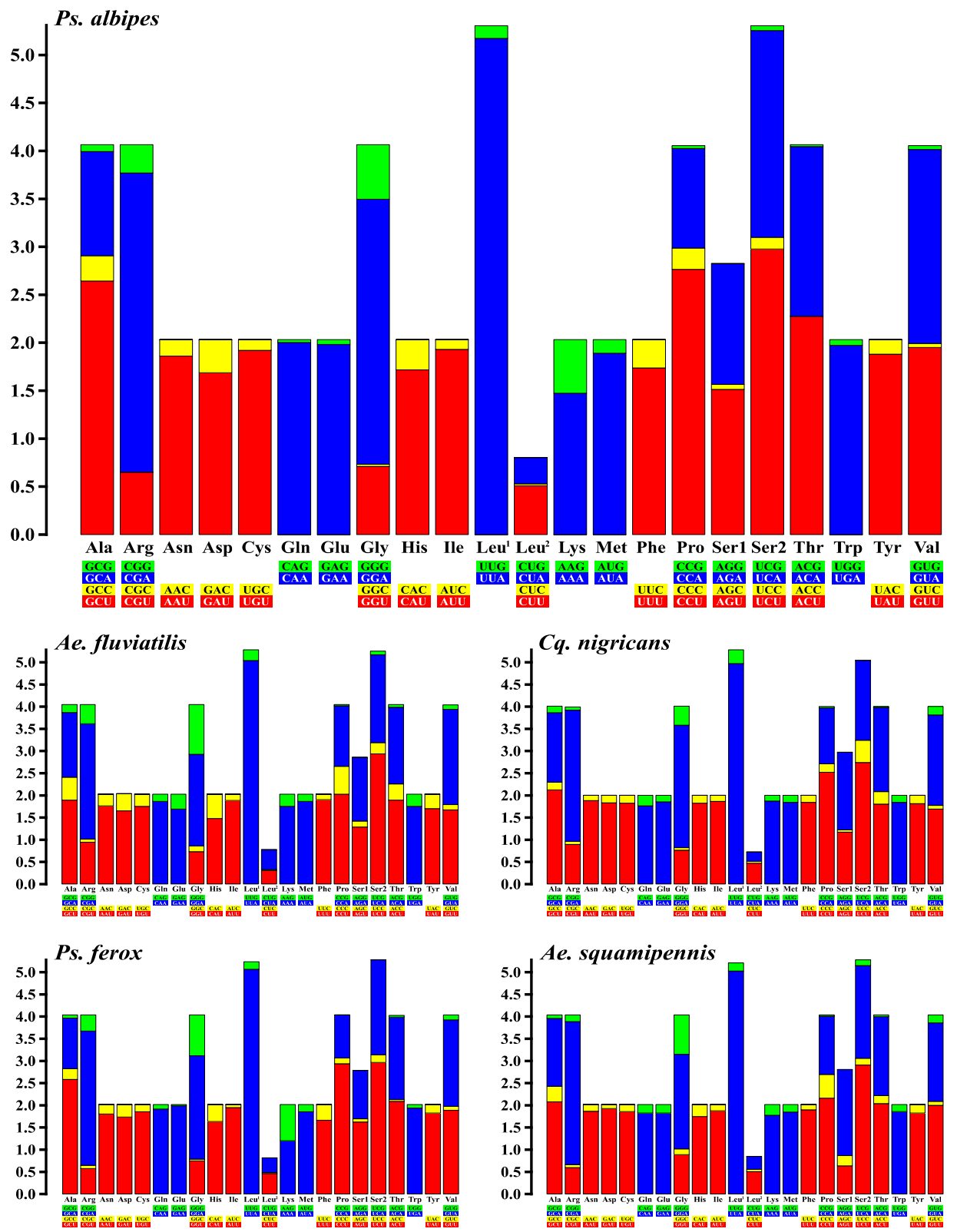

Figure 3. Relative synonymous codon usage (RSCU) of the obtained mitogenomes. RSCU values are represented on the $y$-axis, and families of synonymous codons and their respective amino acids are indicated on the $x$-axis. The synonymous codon families observed in Psorophora albipes (which had its RSCU graph enlarged to make the other graphs easier to read) are the same observed for the other mosquito species. 

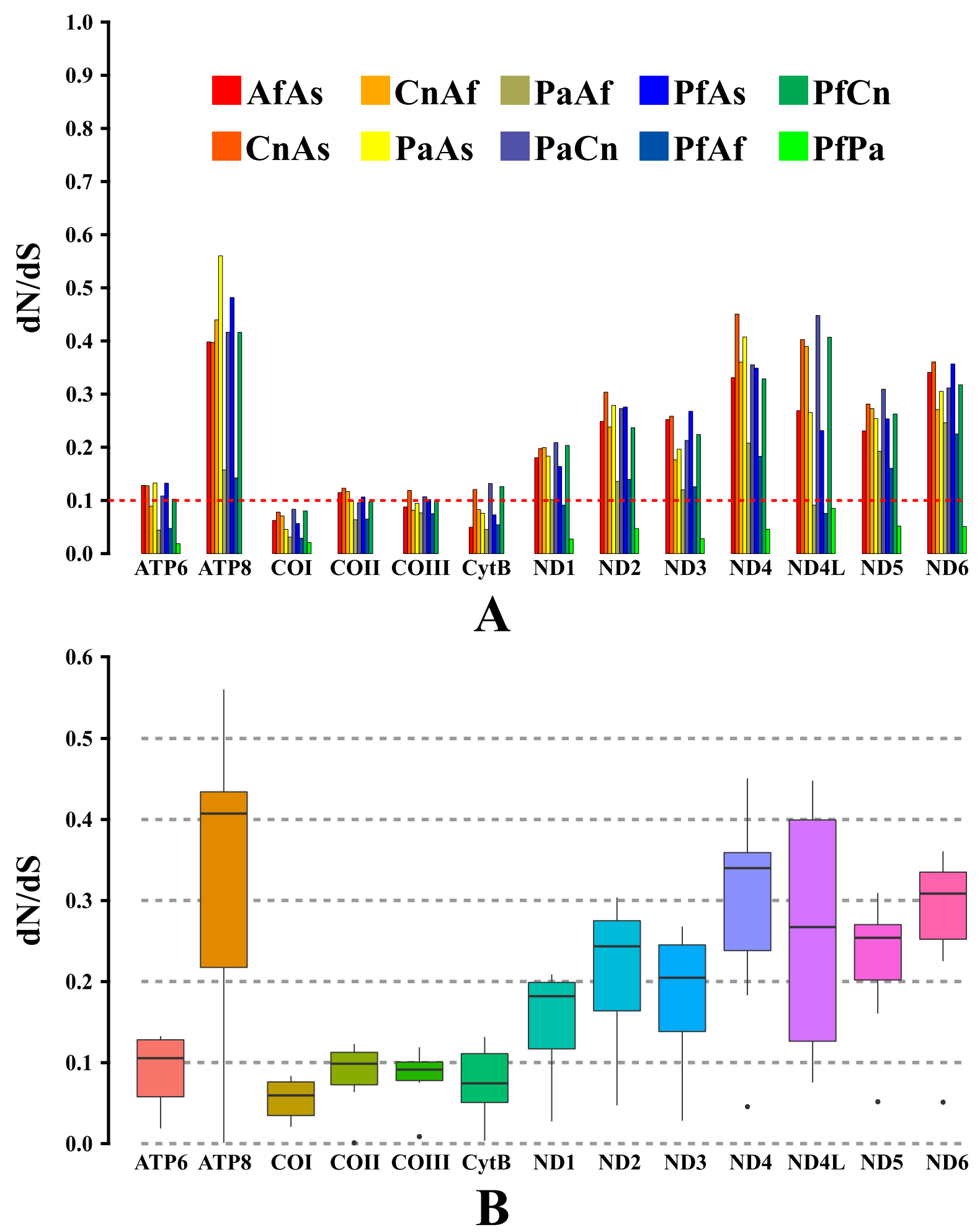

Figure 4. Proportions between rates of non-synonymous $(d N)$ and synonymous $(d S)$ nucleotide substitutions $(d N / d S)$. (A) Bar chart for pairwise proportions of $d N / d S$ for each of the mitochondrial subunits of the investigated species. (B) Box chart illustrating the averages for pairwise proportions of $d N / d S$ for each of the mitochondrial subunits of the investigated species. In the graphs, the $d N / d S$ ratios are plotted on the $y$-axis, and the PCGs are plotted on the $x$-axis.

In addition to the $d N / d S$ analysis, three analyses were performed in order to assess the nucleotide diversity between the mitochondrial sequences obtained in this study, together with other sequences of mosquitoes belonging to the Aedini and Mansoniini tribes (the 
same used for the reconstruction of the phylogenies presented in followed). The first analysis carried out considered the evaluation of nucleotide diversity among mitochondrial sequences of mosquitoes belonging to the Mansoniini tribe; the second analysis considered the evaluation of nucleotide diversity among the mitochondrial sequences of mosquitoes belonging to the Aedini tribe; and the third analysis considered the evaluation of nucleotide diversity among the Ae. squamipennis, the only representative of the Aedeomyiini tribe, together with the others evaluated and belonging to Aedini and Mansoniini. The results showed values of nucleotide diversity $(\pi)$, considering the groups of evaluated sequences, ranging from $0.03 \pm 0.3$ in Mansoniini (Figure 5, blue line); $0.012 \pm 0.128$ in Aedini (Figure 5, red line); and $0.019 \pm 0.118$ evaluating Ae. squamipennis (Aedeomyiini) along with Aedini and Mansoniini (Figure 5, purple line).

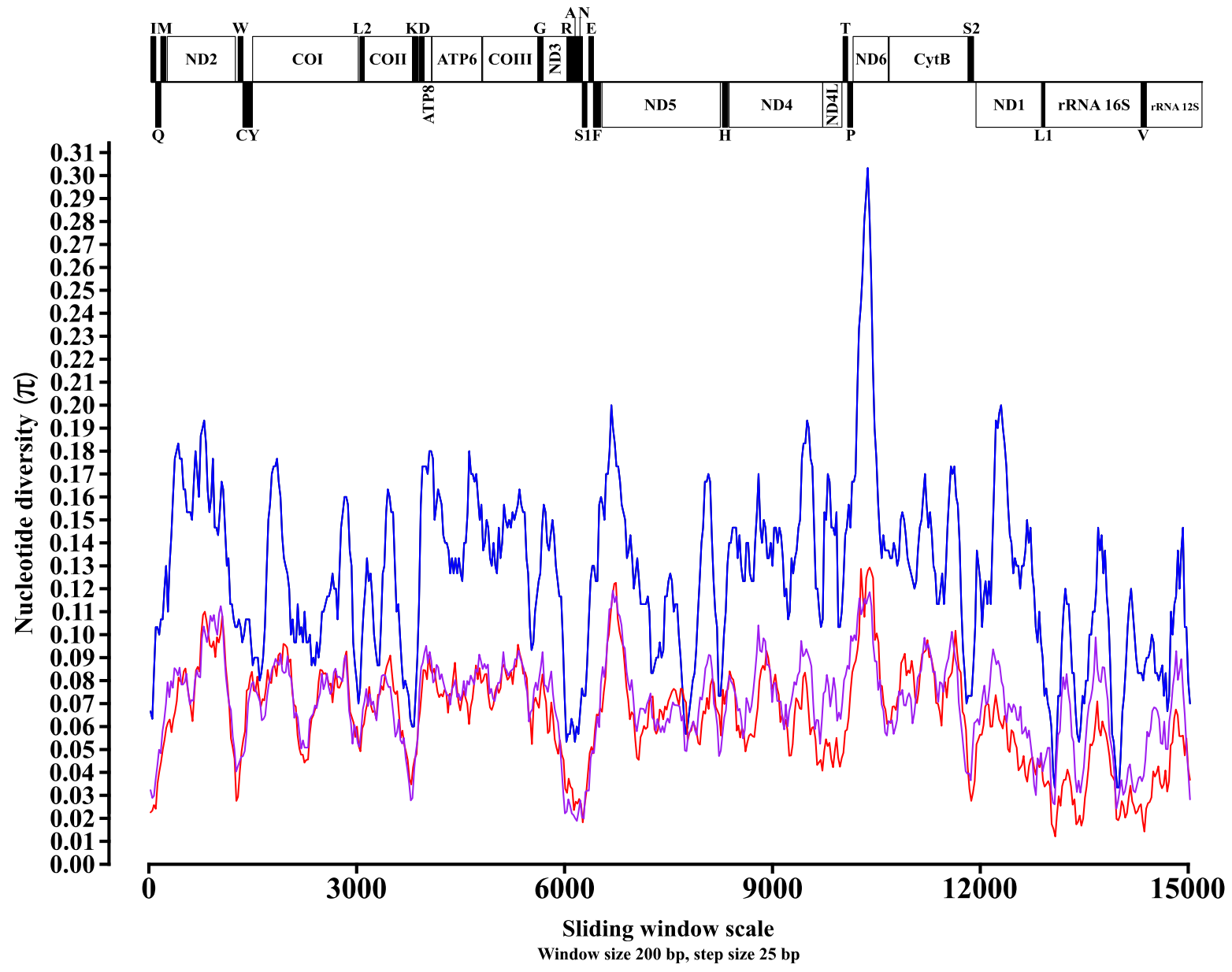

Figure 5. Nucleotide diversity $(\pi)$ among the obtained mitogenomes in this study. Blue lines indicate nucleotide diversity values based on evaluation of sequences from the tribe Mansoniini, including Cq. nigricans. Red lines indicate nucleotide diversity values based on evaluation of sequences from the Aedini tribe, including Ae. fluviatilis, Ps. albipes, and Ps. ferox. The purple lines indicate the nucleotide diversity values based on the evaluation of the Aedeomyia squamipennis (Aedeomyiini) sequence compared to the Mansoniini and Aedini sequence groups. The $\pi$ values were calculated from a $200 \mathrm{bp}$ sliding window analysis in $25 \mathrm{bp}$ steps and are plotted on the $y$-axis. The length values of the aligned sequences of the three evaluated groups are plotted on the $x$-axis. The limits of each gene are indicated in the representation above the graph: vertical black bars indicate tRNAs, and white rectangles indicate PCGs and rRNAs.

The results obtained relate the highest rates of nucleotide diversity in the three groups of sequences evaluated to the regions acting in the protein coding of the analyzed mitogenomes, which it is observed that in at least four of these regions there were sites that reached the maximum diversity scores for each analysis, which are, in order of scoring: ND6, ND5, ND2, and COI. The results obtained, in this way, were similar to other pre- 
viously published ones, when other species of mosquitoes were evaluated [22,23,48,62], highlighting, in addition, the high nucleotide diversity scores for the COI gene, used universally as one of the main molecular markers [63], in both analyses.

In mosquitoes, the proper identification of certain species is difficult due to the great morphological similarity they may present. Therefore, in certain situations, only an observation of internal structures, such as the male specimen's genitalia, allows a reliable identification [64]. In this context, the alternative use of mitochondrial genes, such as COI or $N D 5$, in the identification of cryptic species has shown satisfactory results [14,65-67]. Thus, the results obtained in this study, considering the investigated species, corroborate the use of PCGs as the most suitable regions to be used as molecular markers in the development of evolutionary studies of mosquito species.

\subsection{Phylogenetic Analysis}

The nucleotide sequence alignment using 13 PCGs from 48 taxa (five from this study, the remaining from GenBank and $E M B L$ database), resulted in a nucleotide distance average of $0.16 \%$, the values ranged from $0.03 \%$ to $0.29 \%$ (Table S7).

The two methods of phylogenetic reconstruction (ML and BI) produced identical topologies with high internal anchoring values (Figures 6 and 7), a monophyletic large group included 47 taxa corresponding to the Culicidae family, externally anchored by Dixella aestivalis (Diptera: Dixidae), defined by the automated method of midpoint [68]. The Culicidae family presented a topology with two well-supported clades (BPP $/ \mathrm{BP}=100 \% / 100 \%$ ), corresponding to the subfamilies Anophelinae and Culicinae. The Anophelinae clade, containing 11 species, is external in relation to Culicinae, and evidenced four subclades representing the subgenera Nyssorhynchus, Cellia, Anopheles and Kertezia. Our results corroborate the basal placement of subgenus Kertezia within the genus Anopheles, despite the no representativity of Chagasia genus, that so far is classified as most basal group [69-71]. New evolutionary evidence on temporal diversification has shown that radiation in the Culicinae subfamily is older than Anophelinae and that Bironella phylogenetic status is not stable [72].

The clade that represents the Culicinae subfamily comprises 36 taxa, and was structured into six subclades, representing the tribes Aedini, Culicini, Sabethini, Toxorhynchitini, Mansoniini, and Aedeomyiini (BPP/BP $>98 \%$ ), on this arrangement: Aedeomyiini $+[$ Mansoniini + (Toxorhynchitini + Sabethini $)]+[$ Culicini + Aedini $]$. The Aedeomyiini, represented by the single newly sequenced Ad. squamipennis is placed as the most basal taxa in the Culicinae subfamily anchoring externally all remaining Culinae tribes, strongly supported by the $\mathrm{BPP} / \mathrm{BP}=100 \% / 100 \%$ values. These results differs from those obtained by Reindenbach et al. [11] who placed Aedeomyia + Toxorhynchites as an intermediary taxa, between Sabethini and Aedini tribes in a phylogenetic analysis using six nuclear protein genes. Evolutionary studies with the incomplete mitogenome sequence had clustered Ae. squamipennis with either Uranotaenia or Toxorhynchites genera, but more than that, it suggested that Ad. squamipennis is the earliest divergent taxa in the Culicinae subfamily, with speciation process around 150 million years ago (MYA) [73]. Indeed, the Aedeomyia genus (Theobald, 1901) is promptly distinguished by morphological aspects, and it is thought to be ancient, but extremely specialized taxa that arose in the Old World and spread to some continents, being Ad. squamipennis the single species occurring in the New World. The evolutionary time scale and the geographic boundaries may have contributed to this suggested phylogenetic distance and constitutes an object to further studies [73,74]. Phylogeny inferred from complete mitogenome has producing consistent and more accurate results for evolutionary and taxonomic studies [23], therefore, our results sheds light on the evolutionary history of Aedeomyiini tribe but demands sampling efforts to build a solid molecular taxonomy. 


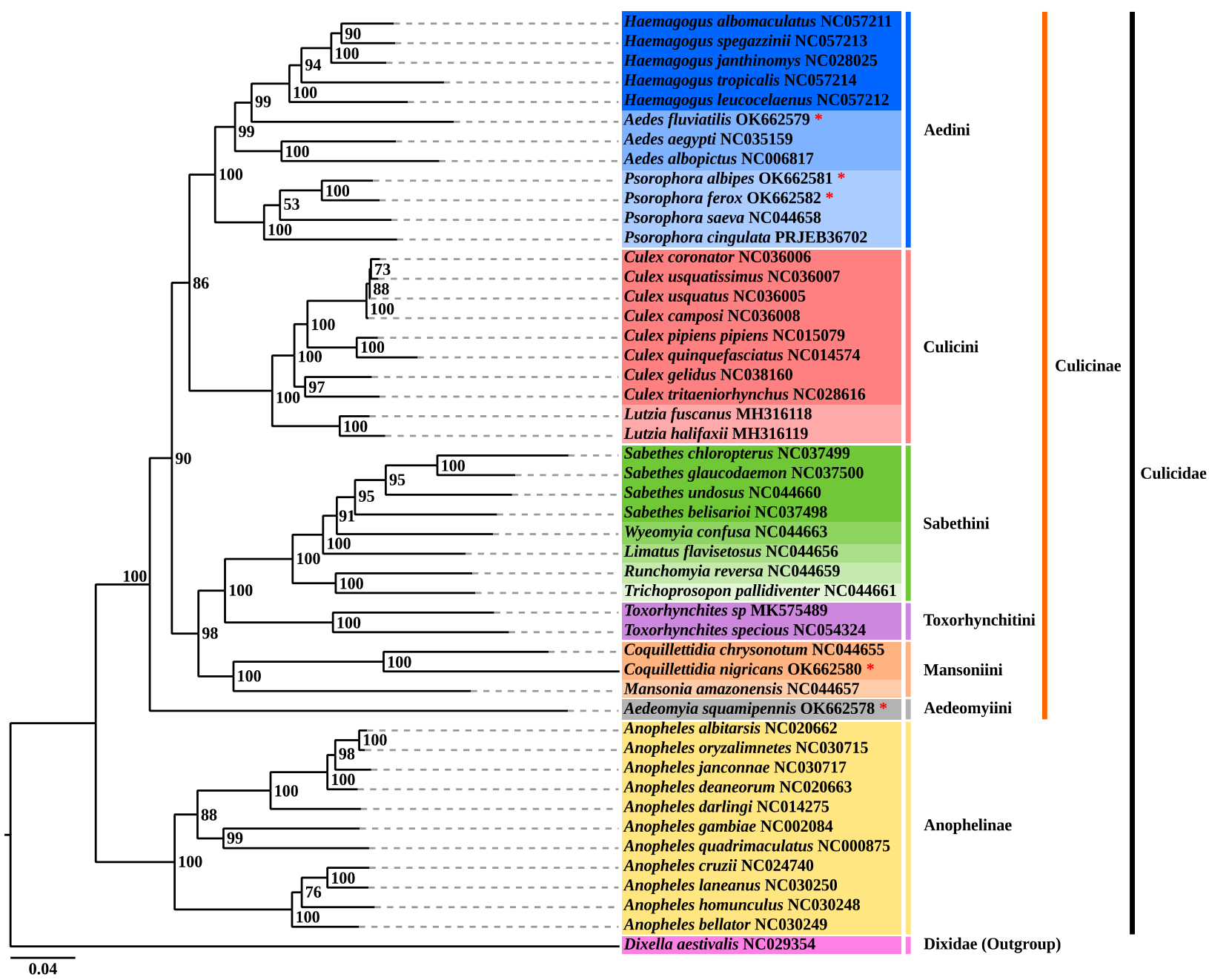

Figure 6. Phylogenetic reconstruction by maximum likelihood based on the 13 PCGs concatenated of the species sequenced in this study (marked with the red asterisk) and 42 other taxa with data available on the GenBank and EMBL databases. The values of support for bootstrapping (BPP) are shown on the left, in each node. Dash lines were drawn for viewing purpose.

The phylogenetic analysis of $C q$. (Rhy.) nigricans resulted in its placement in the Mansoniini tribe (BPP/BP $=100 \% / 100 \%)$ and Coquilletidia genus $(\mathrm{BPP} / \mathrm{BP}=100 \% / 100 \%)$, related to Mansonia genus (BPP/BP $=100 \% / 100 \%)$, in accordance with the current taxonomic classification [75,76]. Regarding the external relationships, our results generate a subclade Mansoniini + [Toxorhynchitini + Sabethini] $($ BPP /BP = 98\%/100\%). Phylogenetic relationships of the Mansoniini are widely accepted to be monophyletic, supported by morphological and bionomic aspects, mainly synapomorphies of immature stages [3]. Moreover, these findings are in accordance with recent evolutionary studies that confirmed the monophyletic status of the tribe, and suggested that Mansoniini is a sister clade of Sabethini tribe that arose nearly 85 MYA [72]. On the other hand, our results contrast with former analysis that related Mansoniini to Aedini tribe, but the non-aedini species were missampling and the target for phylogenetic analysis were different $[11,77]$. 


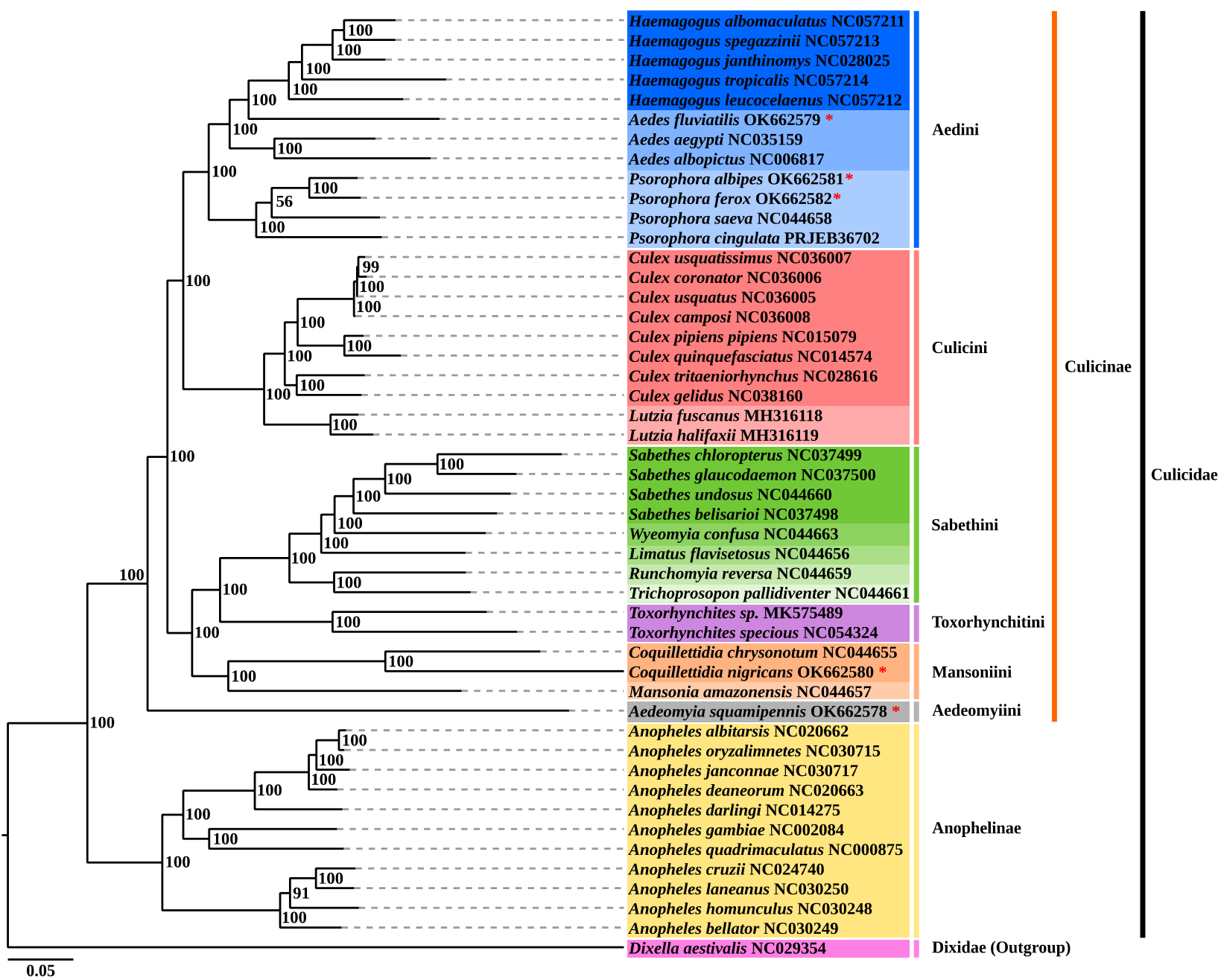

Figure 7. Phylogenetic reconstruction by Bayesian Inference based on the 13 PCGs concatenated of the species sequenced in this study (marked with the red asterisk) and 42 other taxa with data available on the GenBank and EMBL databases. The values of support Bayesian probabilities (BP) are shown on the left, in each node. Dash lines were drawn for viewing purpose.

In this study, three representatives of the Aedini tribe were investigated: Ae. (Grc.) fluviatilis, Ps. (Jan.) albipes, and Ps. (Jan.) ferox. The phylogeny obtained presented a wellsupported clade corresponding to the Aedini tribe (BPP/BP $=100 \% / 100 \%)$. This group included three closely related taxa, corresponding to Haemagogus, Aedes, and Psorophora genera, recovered as sister group to Culicini tribe (BPP/BP $=100 \% / 100 \%$ ), as already suggested by other studies [11,23,72]. Psorophora had basal positioning as already observed by other authors [11,72]. Molecular evolutionary studies suggested a diversification between these tribes around 130 MYA, in the Cretaceous period [72].

The newly sequenced Ae. fluviatilis was placed as a sister group to the Haemagogus clade (BPP $/ \mathrm{BP}=98 \% / 100 \%)$, a topology possibly induced by a missrepresentation of Aedes species. For instance the average of nucleotide distance in tribe level was 0.14 , and very similar values were obtained when compared Ae. fluviatilis to the other genera (Aedes, Psorophora and Haemagogus). Ae. fluviatilis is an anthropophilic mosquito commonly found in urban areas, distributed in the Neotropical region and is considered a potential vector of yellow fever virus. Given its epidemiological potential, recent phylogenetic studies with incomplete mitogenome sequence had found that Ae. fluviatilis is an ancient diverged species regarding the Aedes genus, but with no phyletic stability [72].

The Aedini topology suggests that Ae. fluviatilis is an independent group. In fact there is a strong taxonomic trend that elevates the subgenus Georgecraigius to genus status [78]. 
Massive morphological data were extensively studied and led to this conclusion, therefore, Georgecraigius would comprise subgenera Georgecraigius and Horsfallius, with three species composing [75,79]. In the studies of Reinert, the Georgecraigius genus relates to an Australasian genus, Patmarksia. Later, in order to make Aedini taxonomy treatment more parsimonious, Wilkerson et al. [80] reanalyzed the whole set of morphological data and proposed simplified generic designations, among which Georgecraigius was restored to subgenus level. Aedini is the largest tribe in the Culicidae family and there is an urgent need for much broader studies to reconstruct the natural history of the group.

The topology obtained for the newly sequenced Psorophora species clustered Ps. albipes + Ps. ferox on a subclade that here represents the Janthinosoma subgenus $(\mathrm{BPP} / \mathrm{BP}=$ $100 \% / 100 \%$ ), on the monophyletic genus Psorophora (BPP/BP $=100 \% / 100 \%$ ), and Aedini tribe $(\mathrm{BPP} / \mathrm{BP}=100 \% / 100 \%)$, recovered as sister group to Aedes + Haemagogus clade, as already found by Reinert et al. [75,78,79] and Soghigian et al. [81]. The generic rank retrieved in this analysis for Aedini tribe requires further studies for better resolution, as demonstrated by the Janthinosoma + Psorophora (BPP/BP $=53 \% / 56 \%$ ) subclade. In the study carried out by Silva et al. [72] the incomplete mitogenome sequence of Ps. albipes was assessed and the molecular evolutionary findings clustered Janthinosoma with Grabhamia, but the analysis included only two members of the genus, and no Psorophora (Pso.) species.

Morphological and bionomical aspects were targeted for phylogenetic reconstruction of Psorophora genus in earlier studies [77], and molecular evidence aggregated more value to this taxonomic classification [81]. Nonetheless, the resort of a unique tool, such as morphology, may lead to results that do not always reflect the natural history of the species. Cladistic analysis using morphology recovered the monophyly of the subgenera, although some internal polytomies on the Janthinosoma and Grabhamia were recorded [82].

The investigation over the mitochondrial genome of five species of mosquitoes occurring in the Brazilian Amazon region allowed us to obtain important information about the taxonomy and evolutionary biology of the Culicidae. Classical taxonomy based on the morphology and bionomics of the species is undoubtedly the main path to classification process, nevertheless, such method has its flaws, especially when it comes to a taxa as diverse and ancient as the Culicidae family: morphological and bionomical characteristics may arise and vanish in the same group over time. The advent of molecular tools has allowing deeper investigation on the taxonomic relationships between these organisms, recent studies has providing knowledge on the natural relatedness, biogeographical and ancestry. In this sense, it is important to highlight that mitochondrial characterization studies and the consequent inclusion of more Culicidae taxa in public databases will support more comprehensive phylogenetic reconstruction analyses and elucidate taxonomic proposals.

\section{Conclusions}

The aim of this study was to enrich knowledge on the molecular aspects and to propose a phylogenetic reconstruction of the Culicidae family based on mtDNA. This is the first description of the complete mitochondrial DNA of Ae. (Grg.) fluviatilis, Ad. squaminpennis, Cq. (Rhy.) nigricans, Ps. (Jan.) albipes and Ps. (Jan.) ferox. All mitogenomes evaluated were similar to the mtDNA molecule pattern for Culicidae family: 37 subunits subdivided in 13 PCGs, two rRNAs and 22 tRNAs. The analyses of the individual genes showed that COI is the most preserved subunit and more suitable for taxonomic purposes and ATP8 is the less conservative gene, the 13 PCGs combined demonstrated to be a more complete object of study for both taxonomic and phylogenetic analyses. ML and BI inference yielded identical topologies where $A d$. squamipennis was placed as the most external lineage from Culicinae subfamily; $C q$. nigricans related to Cq. chrysonotum, both related to Mansonia; Ae. fluviatilis positioned in Aedini tribe but as a sister clade to Aedes (Stg.) spp. which clearly demonstrated the necessity for more representative studies within the genus; both Ps. albipes and Ps. ferox clustered together as the Janthinosoma group on Psorophora subclade, this the first analysis with complete mtDNA emcompassing all subgenera of the genus. All phylogenetic results confirm the current taxonomic proposition for the studied tribes. 
These finding will support future hypotheses on taxonomic and evolutionary traits for the Culicidae family.

Supplementary Materials: The following content are available online at https:/ / www.mdpi.com/ article/10.3390 / genes12121983/s1, Figure S1: Secondary structures of Ps. albipes tRNAs, Figure S2: Secondary structures of Ps. ferox tRNAs, Figure S3: Secondary structures of Ae. fluviatilis tRNAs, Figure S4: Secondary structures of Cq. nigricans tRNAs, Figure S5: Secondary structures of $A d$. squamipennis tRNAs, Table S1: Nucleotide composition of the mtDNA of Ps. albipes, Table S2: Nucleotide composition of the mtDNA of Ps. ferox, Table S3: Nucleotide composition of the mtDNA of Ae. fluviatilis, Table S4: Nucleotide composition of the mtDNA of Cq. nigricans, Table S5: Nucleotide composition of the mtDNA of Ad. squamipennis, Table S6: RSCU metrics of obtained sequences, Table S7: Nucleotide distance matrix of taxa used in phylogeny reconstruction analysis.

Author Contributions: B.L.S.d.N. and F.S.d.S. contributed equally to this paper. B.L.S.d.N., J.P.N.-N. and F.S.d.S. designed the study. F.S.d.S., B.L.S.d.N., D.B.d.A.M. and S.P.d.S. designed the methodology. B.L.S.d.N., L.H.d.S.e.S., H.A.d.O.M., D.D.D., D.B.R.V., J.W.R.J. and R.C.F.B. collected biological samples. J.P.N.-N., D.B.d.A.M., A.C.R.C., J.O.C. and L.C.M. provided laboratory resources. S.P.d.S. and B.L.S.d.N. prepared genomic library and conducted NGS sequencing. F.S.d.S. performed bioinformatic analysis. B.L.S.d.N. and F.S.d.S. wrote the first draft of the manuscript. J.P.N.-N., D.B.d.A.M., A.C.R.C. and P.F.d.C.V. supervised the study. J.P.N.-N. and P.F.d.C.V. managed the project. All authors have read and agreed to the published version of the manuscript.

Funding: The APC was funded by Evandro Chagas Institute-IEC/SVS/MS.

Data Availability Statement: All data generated during this study are available as tables and figures included in this published article and its Supplementary Information files. The GenBank database accession numbers for the four mitochondrial genomes sequenced in this study are OK662581 (Ps. albipes), OK662582 (Ps. ferox), OK662579 (Ae. fluviatilis), OK662580 (Cq. nigricans), and OK662578 (Ad. squamipennis).

Conflicts of Interest: The authors declare no conflict of interest.

\section{References}

1. Harbach, R.E. Mosquito Taxonomic Inventory. 2013. Available online: https://www.mosquito-taxonomic-inventory.info/ (accessed on 3 November 2021).

2. Harbach, R.E. The Culicidae (Diptera): A Review of Taxonomy, Classification and Phylogeny. Zootaxa 2007, 1668, 591-638. [CrossRef]

3. Harbach, R.E.; Kitching, I.J. Phylogeny and Classification of the Culicidae (Diptera). Syst. Entomol. 1998, 23, 327-370. [CrossRef]

4. Forattini, O.P. Culicidologia Médica: Identificação, Biologia, Epidemiologia; EDUSP: São Paulo, Brazil, 2002.

5. Hao, Y.J.; Zou, Y.L.; Ding, Y.R.; Xu, W.Y.; Yan, Z.T.; Li, X.D.; Fu, W.B.; Li, T.J.; Chen, B. Complete Mitochondrial Genomes of Anopheles stephensi and An. dirus and Comparative Evolutionary Mitochondriomics of 50 Mosquitoes. Sci. Rep. 2017, 7, 7666. [CrossRef]

6. Sallum, M.A.M.; Schultz, T.R.; Foster, P.G.; Aronstein, K.; Wirtz, R.A.; Wilkerson, R.C. Phylogeny of Anophelinae (Diptera: Culicidae) Based on Nuclear Ribosomal and Mitochondrial DNA Sequences. Syst. Entomol. 2002, 27, 361-382. [CrossRef]

7. Hay, S.I.; Sinka, M.E.; Okara, R.M.; Kabaria, C.W.; Mbithi, P.M.; Tago, C.C.; Benz, D.; Gething, P.W.; Howes, R.E.; Patil, A.P.; et al. Developing Global Maps of the Dominant Anopheles Vectors of Human Malaria. PLoS Med. 2010, 7, e1000209. [CrossRef] [PubMed]

8. Nanfack Minkeu, F.; Vernick, K.D. A Systematic Review of the Natural Virome of Anopheles mosquitoes. Viruses 2018, 10, 222. [CrossRef] [PubMed]

9. Foster, P.G.; De Oliveira, T.M.P.; Bergo, E.S.; Conn, J.; Sant'Ana, D.C.; Nagaki, S.S.; Nihei, S.; Lamas, C.E.; Gonzalez, C.; Moreira, C.C.; et al. Phylogeny of Anophelinae Using Mitochondrial Protein Coding Genes. R. Soc. Open Sci. 2017, 4, 170758. [CrossRef] [PubMed]

10. Judd, D.D. Review of the Systematics and Phylogenetic Relationships of the Sabethini (Diptera: Culicidae). Syst. Entomol. 1996, 21, 129-150. [CrossRef]

11. Reidenbach, K.R.; Cook, S.; Bertone, M.A.; Harbach, R.E.; Wiegmann, B.M.; Besansky, N.J. Phylogenetic Analysis and Temporal Diversification of Mosquitoes (Diptera: Culicidae) Based on Nuclear Genes and Morphology. BMC Evol. Biol. 2009, 9, 298. [CrossRef]

12. Lorenz, C.; Alves, J.M.P.; Foster, P.G.; Sallum, M.A.M.; Suesdek, L. First Record of Translocation in Culicidae (Diptera) Mitogenomes: Evidence from the Tribe Sabethini. BMC Genom. 2019, 20, 721. [CrossRef] [PubMed] 
13. Laurito, M.; de Oliveira, T.M.P.; Almirón, W.R.; Sallum, M.A.M. COI Barcode versus Morphological Identification of Culex (Culex) (Diptera: Culicidae) Species: A Case Study Using Samples from Argentina and Brazil. Mem. Inst. Oswaldo Cruz 2013, 108, 110-122. [CrossRef] [PubMed]

14. Laurito, M.; Ayala, A.M.; Almirón, W.R.; Gardenal, C.N. Molecular Identification of Two Culex (Culex) Species of the Neotropical Region (Diptera: Culicidae). PLoS ONE 2017, 12, e0173052. [CrossRef]

15. Chan-Chable, R.J.; Martínez-Arce, A.; Mis-Avila, P.C.; Ortega-Morales, A.I. DNA Barcodes and Evidence of Cryptic Diversity of Anthropophagous Mosquitoes in Quintana Roo, Mexico. Ecol. Evol. 2019, 9, 4692-4705. [CrossRef]

16. DeSalle, R.; Egan, M.G.; Siddall, M. The Unholy Trinity: Taxonomy, Species Delimitation and DNA Barcoding. Philos. Trans. R. Soc. B Biol. Sci. 2005, 360, 1905-1916. [CrossRef]

17. Tabachnick, W.J. Nature, Nurture and Evolution of Intra-Species Variation in Mosquito Arbovirus Transmission Competence. Int. J. Environ. Res. Public Health 2013, 10, 249-277. [CrossRef]

18. Barzon, L.; Lavezzo, E.; Militello, V.; Toppo, S.; Palù, G. Applications of Next-Generation Sequencing Technologies to Diagnostic Virology. Int. J. Mol. Sci. 2011, 12, 7861-7884. [CrossRef]

19. Boore, J.L. Animal Mitochondrial Genomes. Nucleic Acids Res. 1999, 27, 1767-1780. [CrossRef]

20. Aragão, A.O.; Nunes Neto, J.P.; Cruz, A.C.R.; Casseb, S.M.M.; Cardoso, J.F.; da Silva, S.P.; Ishikawa, E.A.Y. Description and Phylogeny of the Mitochondrial Genome of Sabethes chloropterus, Sabethes glaucodaemon and Sabethes belisarioi (Diptera: Culicidae). Genomics 2019, 111, 607-611. [CrossRef] [PubMed]

21. Chen, K.; Wang, Y.; Li, X.Y.; Peng, H.; Ma, Y.J. Sequencing and Analysis of the Complete Mitochondrial Genome in Anopheles sinensis (Diptera: Culicidae). Infect. Dis. Poverty 2017, 6, 1-6. [CrossRef] [PubMed]

22. Demari-Silva, B.; Foster, P.G.; de Oliveira, T.M.P.; Bergo, E.S.; Sanabani, S.S.; Pessôa, R.; Sallum, M.A.M. Mitochondrial Genomes and Comparative Analyses of Culex camposi, Culex coronator, Culex usquatus and Culex usquatissimus (Diptera:CUlicidae), Members of the Coronator Group. BMC Genom. 2015, 16, 831. [CrossRef]

23. Da Silva, F.S.; Cruz, A.C.R.; de Almeida Medeiros, D.B.; Silva, S.P.; Nunes, M.R.T.; Martins, L.C.; Chiang, J.O.; da Silva Lemos, P.; Cunha, G.M.; Araujo, R.F.; et al. Mitochondrial Genome Sequencing and Phylogeny of Haemagogus albomaculatus, Haemagogus leucocelaenus, Haemagogus spegazzinii, and Haemagogus tropicalis (Diptera: Culicidae). Sci. Rep. 2020, 10, 16948. [CrossRef]

24. Ye, F.; Liu, T.; King, S.D.; You, P. Mitochondrial Genomes of Two Phlebotomine Sand Flies, Phlebotomus chinensis and Phlebotomus papatasi (Diptera: Nematocera), the First Representatives from the Family Psychodidae. Parasites Vectors 2015, 8, 1-13. [CrossRef] [PubMed]

25. Song, N.; Liang, A.P.; Bu, C.P. A Molecular Phylogeny of Hemiptera Inferred from Mitochondrial Genome Sequences. PLoS ONE 2012, 7, e48778. [CrossRef] [PubMed]

26. Pita, S.; Panzera, F.; Vela, J.; Mora, P.; Palomeque, T.; Lorite, P. Complete Mitochondrial Genome of Triatoma Infestans (Hemiptera, Reduviidae, Triatominae), Main Vector of Chagas Disease. Infect. Genet. Evol. 2017, 54, 158-163. [CrossRef] [PubMed]

27. Andrews, S. FastQC v.0.11.9. Available online: https://www.bioinformatics.babraham.ac.uk/projects/fastqc/ (accessed on 16 September 2021).

28. Krueger, F. Trim Galore v.0.6.5. Available online: https://www.bioinformatics.babraham.ac.uk/projects/trim_galore/ (accessed on 16 September 2021).

29. Li, D.; Liu, C.-M.; Luo, R.; Sadakane, K.; Lam, T.-W. MEGAHIT: An Ultra-Fast Single-Node Solution for Large and Com- Plex Metagenomics Assembly via Succinct de Bruijn Graph. Bioinformatics 2015, 31, 1674-1676. [CrossRef]

30. Bankevich, A.; Nurk, S.; Antipov, D.; Gurevich, A.A.; Dvorkin, M.; Kulikov, A.S.; Lesin, V.M.; Nikolenko, S.I.; Pham, S.; Prjibelski, A.D.; et al. SPAdes: A New Genome Assembly Algorithm and Its Applications to Single-Cell Sequencing. J. Comput. Biol. 2012, 19, 455-477. [CrossRef]

31. Buchfink, B.; Xie, C.; Huson, D.H. Fast and Sensitive Protein Alignment Using DIAMOND. Nat. Methods 2014, 12, 59-60. [CrossRef]

32. Huson, D.H.; Auch, A.F.; Qi, J.; Schuster, S.C. MEGAN Analysis of Metagenomic Data. Genome Res. 2007, 17, 377-386. [CrossRef]

33. Kearse, M.; Moir, R.; Wilson, A.; Stones-Havas, S.; Cheung, M.; Sturrock, S.; Buxton, S.; Cooper, A.; Markowitz, S.; Duran, C.; et al. Geneious Basic: An Integrated and Extendable Desktop Software Platform for the Organization and Analysis of Sequence Data. Bioinformatics 2012, 28, 1647-1649. [CrossRef]

34. Bernt, M.; Donath, A.; Jühling, F.; Externbrink, F.; Florentz, C.; Fritzsch, G.; Pütz, J.; Middendorf, M.; Stadler, P.F. MITOS: Improved de Novo Metazoan Mitochondrial Genome Annotation. Mol. Phylogenet. Evol. 2013, 69, 313-319. [CrossRef]

35. Stothard, P.; Wishart, D.S. Circular Genome Visualization and Exploration Using CGView. Bioinformatics 2005, 21, 537-539. [CrossRef]

36. Kumar, S.; Stecher, G.; Li, M.; Knyaz, C.; Tamura, K. MEGA X: Molecular Evolutionary Genetics Analysis across Computing Platforms. Mol. Biol. Evol. 2018, 35, 1547-1549. [CrossRef]

37. Perna, N.T.; Kocher, T.D. Patterns of Nucleotide Composition at Fourfold Degenerate Sites of Animal Mitochondrial Genomes. J. Mol. Evol. 1995, 41, 353-358. [CrossRef] [PubMed]

38. Pfeifer, B.; Wittelsbürger, U.; Ramos-Onsins, S.E.; Lercher, M.J. PopGenome: An Efficient Swiss Army Knife for Population Genomic Analyses in R. Mol. Biol. Evol. 2014, 31, 1929-1936. [CrossRef] [PubMed]

39. R Core Team. R: A Language and Environment for Statistical Computing; R Foundation for Statistical Computing: Vienna, Austria, 2020. 
40. Yang, Z. PAML 4: Phylogenetic Analysis by Maximum Likelihood. Mol. Biol. Evol. 2007, 24, 1586-1591. [CrossRef] [PubMed]

41. Katoh, K.; Standley, D.M. MAFFT Multiple Sequence Alignment Software Version 7: Improvements in Performance and Usability. Mol. Biol. Evol. 2013, 30, 772-780. [CrossRef]

42. Nguyen, L.T.; Schmidt, H.A.; Von Haeseler, A.; Minh, B.Q. IQ-TREE: A Fast and Effective Stochastic Algorithm for Estimating Maximum-Likelihood Phylogenies. Mol. Biol. Evol. 2015, 32, 268-274. [CrossRef]

43. Huelsenbeck, J.P.; Ronquist, F. MRBAYES: Bayesian Inference of Phylogenetic Trees. Bioinformatics 2001, 17, 754-755. [CrossRef]

44. Rambaut, A. Figtree v1.4.4. Available online: http://tree.bio.ed.ac.uk/software/figtree/ (accessed on 1 November 2021).

45. Inkscape. Available online: https://inkscape.org/pt-br/ (accessed on 1 November 2021).

46. Sun, L.; Li, T.J.; Fu, W.B.; Yan, Z.T.; Si, F.L.; Zhang, Y.J.; Mao, Q.M.; Demari-Silva, B.; Chen, B. The Complete Mt Genomes of Lutzia halifaxia, Lt. fuscanus and Culex pallidothorax (Diptera: Culicidae) and Comparative Analysis of 16 Culex and Lutzia Mt Genome Sequences. Parasites Vectors 2019, 12,1-13. [CrossRef] [PubMed]

47. Martinez-Villegas, L.; Assis-Geraldo, J.; Koerich, L.B.; Collier, T.C.; Lee, Y.; Main, B.J.; Rodrigues, N.B.; Orfano, A.S.; Pires, A.C.A.M.; Campolina, T.B.; et al. Characterization of the Complete Mitogenome of Anopheles Aquasalis, and Phylogenetic Divergences among Anopheles from Diverse Geographic Zones. PLoS ONE 2019, 14, e219523. [CrossRef]

48. Luo, Q.C.; Hao, Y.J.; Meng, F.; Li, T.J.; Ding, Y.R.; Hua, Y.Q.; Chen, B. The Mitochondrial Genomes of Culex tritaeniorhynchus and Culex pipiens Pallens (Diptera: Culicidae) and Comparison Analysis with Two Other Culex Species. Parasites Vectors 2016, 9, 406. [CrossRef] [PubMed]

49. Zhang, D.X.; Hewitt, G.M. Insect Mitochondrial Control Region: A Review of Its Structure, Evolution and Usefulness in Evolutionary Studies. Biochem. Syst. Ecol. 1997, 25, 99-120. [CrossRef]

50. Zhang, S.K.; Shu, J.P.; Wang, Y.D.; Liu, Y.N.; Peng, H.; Zhang, W.; Wang, H.J. The Complete Mitochondrial Genomes of Two Sibling Species of Camellia weevils (Coleoptera: Curculionidae) and Patterns of Curculionini Speciation. Sci. Rep. 2019 , 9, 3412. [CrossRef] [PubMed]

51. Behura, S.K.; Lobo, N.F.; Haas, B.; deBruyn, B.; Lovin, D.D.; Shumway, M.F.; Puiu, D.; Romero-Severson, J.; Nene, V.; Severson, D.W. Complete Sequences of Mitochondria Genomes of Aedes aegypti and Culex quinquefasciatus and Comparative Analysis of Mitochondrial DNA Fragments Inserted in the Nuclear Genomes. Insect Biochem. Mol. Biol. 2011, 41, 770-777. [CrossRef] [PubMed]

52. Oliveira, T.M.P.; Foster, P.G.; Bergo, E.S.; Nagaki, S.S.; Sanabani, S.S.; Marinotti, O.; Marinotti, P.N.; Sallum, M.A.M. Mitochondrial Genomes of Anopheles (Kerteszia) (Diptera: Culicidae) from the Atlantic Forest, Brazil. J. Med. Entomol. 2016, 53, 790-797. [CrossRef]

53. Timmis, J.N.; Ayliff, M.A.; Huang, C.Y.; Martin, W. Endosymbiotic Gene Transfer: Organelle Genomes Forge Eukaryotic Chromosomes. Nat. Rev. Genet. 2004, 5, 123-135. [CrossRef]

54. Lemos, P.d.S.; Monteiro, H.A.d.O.; Castro, F.C.; de Lima, C.P.S.; da Silva, D.E.A.; de Vasconcelos, J.M.; de Oliveira, L.F.; da Silva, S.P.; Cardoso, J.F.; Vianez Júnior, J.L.d.S.G.; et al. Characterization of Mitochondrial Genome of Haemagogus janthinomys (Diptera: Culicidae). Mitochondrial DNA Part A DNA Mapp. Seq. Anal. 2017, 28, 50-51. [CrossRef] [PubMed]

55. Kambhampati, S.; Smith, P.T. PCR Primers for the Amplification of Four Insect Mitochondrial Gene Fragments. Insect Mol. Biol. 1995, 4, 233-236. [CrossRef]

56. Xu, J.; Fonseca, D.M. One-Way Sequencing of Multiple Amplicons from Tandem Repetitive Mitochondrial DNA Control Region. Mitochondrial DNA 2011, 22, 155-158. [CrossRef]

57. Wang, G.; Li, C.; Guo, X.; Xing, D.; Dong, Y.; Wang, Z.; Zhang, Y.; Liu, M.; Zheng, Z.; Zhang, H.; et al. Identifying the Main Mosquito Species in China Based on DNA Barcoding. PLoS ONE 2012, 7, e47051. [CrossRef] [PubMed]

58. Makhawi, A.M.; Liu, X.B.; Yang, S.R.; Liu, Q.Y. Genetic Variations of ND5 Gene of MtDNA in Populations of Anopheles sinensis (Diptera: Culicidae) Malaria Vector in China. Parasites Vectors 2013, 6, 1-11. [CrossRef]

59. Pu, D.Q.; Liu, H.L.; Gong, Y.Y.; Ji, P.C.; Li, Y.J.; Mou, F.S.; Wei, S.J. Mitochondrial Genomes of the Hoverflies Episyrphus balteatus and Eupeodes corollae (Diptera: Syrphidae), with a Phylogenetic Analysis of Muscomorpha. Sci. Rep. 2017, 7, 44300. [CrossRef] [PubMed]

60. Li, J.; Zhang, Y.; Hu, K.; Zhao, Y.; Lin, R.; Li, Y.; Huang, Z.; Zhang, X.; Geng, X.; Ding, J. Mitochondrial Genome Characteristics of Two Sphingidae Insects (Psilogramma increta and Macroglossum stellatarum) and Implications for Their Phylogeny. Int. J. Biol. Macromol. 2018, 113, 592-600. [CrossRef] [PubMed]

61. Wang, J.J.; Li, D.F.; Li, H.; Yang, M.F.; Dai, R.H. Structural and Phylogenetic Implications of the Complete Mitochondrial Genome of Ledra Auditura. Sci. Rep. 2019, 9, 15746. [CrossRef]

62. Krzywinski, J.; Li, C.; Morris, M.; Conn, J.E.; Lima, J.B.; Povoa, M.M.; Wilkerson, R.C. Analysis of the Evolutionary Forces Shaping Mitochondrial Genomes of a Neotropical Malaria Vector Complex. Mol. Phylogenet. Evol. 2011, 58, 469-477. [CrossRef] [PubMed]

63. Hebert, P.D.N.; Cywinska, A.; Ball, S.L.; DeWaard, J.R. Biological Identifications through DNA Barcodes. Proc. R. Soc. B Biol. Sci. 2003, 270, 313-321. [CrossRef] [PubMed]

64. Laurito, M.; Almirón, W.R. Phylogenetic Relationships of Culex (Culex) Species (Diptera, Culicidae) from Argentina Based on Morphological Characters. Zootaxa 2013, 3652, 117-155. [CrossRef] [PubMed]

65. Cywinska, A.; Hunter, F.F.; Hebert, P.D.N. Identifying Canadian Mosquito Species through DNA Barcodes. Med. Vet. Entomol. 2006, 20, 413-424. [CrossRef] [PubMed] 
66. Murugan, K.; Vadivalagan, C.; Karthika, P.; Panneerselvam, C.; Paulpandi, M.; Subramaniam, J.; Wei, H.; Aziz, A.T.; Alsalhi, M.S.; Devanesan, S.; et al. DNA Barcoding and Molecular Evolution of Mosquito Vectors of Medical and Veterinary Importance. Parasitol. Res. 2016, 115, 107-121. [CrossRef] [PubMed]

67. Gonz, R. Molecular Tracing with Mitochondrial ND5 of the Invasive Mosquito Aedes (Stegomyia) Albopictus (Skuse) in Northern South America. J. Entomol. Zool. Stud. 2016, 1, 32-39.

68. Hess, P.N.; De Moraes Russo, C.A. An Empirical Test of the Midpoint Rooting Method. Biol. J. Linn. Soc. 2007, 92, 669-674. [CrossRef] [PubMed]

69. Harbach, R.E.; Kitching, I.J. The Phylogeny of Anophelinae Revisited: Inferences about the Origin and Classification of Anopheles (Diptera: Culicidae). Zool. Scr. 2016, 45, 34-47. [CrossRef]

70. Harbach, R.E. The Phylogeny and Classification of Anopheles. In Anopheles mosquitoes—New Insights into Malaria Vectors, Sylvie Manguin; InTech: Rijeka, Croatia, 2013. [CrossRef]

71. Peng, X.Y.; Zhou, P.; Duan, X.Y.; Qian, Z.Q. The Mitochondrial Genomes of Twelve Anopheles mosquitoes (Diptera: Culicidae) and Their Phylogenetic Implications. Conserv. Genet. Resour. 2016, 8, 387-390. [CrossRef]

72. Silva, A.F.; Machado, L.C.; de Paula, M.B.; da Silva Pessoa Vieira, C.J.; de Morais Bronzoni, R.V.; de Melo Santos, M.A.V.; Wallau, G.L. Culicidae Evolutionary History Focusing on the Culicinae Subfamily Based on Mitochondrial Phylogenomics. Sci. Rep. 2020, 10, 18823. [CrossRef]

73. Tyson, W.H. Contributions to the Mosquito Fauna of Southeast Asia. VII. Genus Aedeomyia Theobald in South-East Asia. Contrib. Am. Entomol. Inst. 1970, 6, 1-27.

74. Belkin, J.N. The Mosquitoes of the South Pacific (Diptera, Culicidae); Cambridge University Press: London, UK, $1962 ;$ Volume 2.

75. Reinert, J.F. List of Abbreviations for Currently Valid Generic-Level Taxa in Family Culicidae (Diptera). J. Eur. Mosq. Control Assoc. 2009, 27, 68-76.

76. Reinert, J.F. List of Species in Tribe Mansoniini with Published Illustrations and/or Descriptions of Eggs (Diptera: Culicidae). Eur. Mosq. Bull. 2010, 28, 143-147.

77. Reinert, J.F.; Harbach, R.E.; Kitching, I.J. Phylogeny and Classification of Tribe aedini (Diptera: Culicidae). Zool. J. Linn. Soc. 2009, 157, 700-794. [CrossRef]

78. Reinert, J.F.; Harbach, R.E.; Kitching, I.J. Phylogeny and Classification of Aedini (Diptera: Culicidae), Based on Morphological Characters of All Life Stages. Zool. J. Linn. Soc. 2004, 142, 289-368. [CrossRef]

79. Reinert, J.F. Comparative Anatomy of the Female Genitalia of Generic-Level Taxa in Tribe aedini (Diptera: Culicidae). Part XV. Genus Georgecraigius Reinert, Harbach and Kitching. Contrib. Am. Entomol. Inst. 2008, 35, 1-10.

80. Wilkerson, R.C.; Linton, Y.M.; Fonseca, D.M.; Schultz, T.R.; Price, D.C.; Strickman, D.A. Making Mosquito Taxonomy Useful: A Stable Classification of Tribe Aedini That Balances Utility with Current Knowledge of Evolutionary Relationships. PLoS ONE 2015, 10, e133602. [CrossRef]

81. Soghigian, J.; Andreadis, T.G.; Livdahl, T.P. From ground pools to treeholes: Convergent evolution of habitat and phenotype in Aedes mosquitoes. BMC Evol. Biol. 2017, 17, 262. [CrossRef] [PubMed]

82. Liria, J.; Navarro, J.-C. Phylogenetic Relationships in the Genus Psorophora Robineau-Desvoidy (Diptera: Culicidae), Based on Morphological Characters. Entomol. Appl. Sci. Lett. 2014, 4, 22-50. 